\title{
A FLAT SHIP THEORY ON BOW AND STERN FLOWS
}

\author{
SONGPING ZHU' ${ }^{1}$ and YINGLONG ZHANG ${ }^{2}$
}

(Received 18 December, 2000; revised 9 September, 2002)

\begin{abstract}
An analytical solution of a two-dimensional bow and stern flow model based on a flat ship theory is presented for the first time. The flat ship theory is a counterpart to Michell's thin ship theory and leads to a mixed initial-boundary value problem, which is usually difficult to solve analytically. Starting from the transient problem, we shall first show that a steady state is attainable at the large time limit. Then the steady problem is solved in detail by means of the Wiener-Hopf technique and closed-form far-field results are obtained for an arbitrary hull shape. A part from providing a better understanding of the underlying physics, the newly found analytical solution has shed some light on solving a longtime outstanding problem in the engineering practice of ship building, the optimisation of hull shape.
\end{abstract}

\section{Introduction}

Free-surface flow past a surface-piercing object such as a ship is a very important yet challenging problem. Two widely used models are Michell's thin ship theory $([17,23,24])$ and the flat ship theory proposed later as a parallel model to the thin ship theory $([2,16,25])$. It should be noted that thin ship theory has no applications in two-dimensional ship problems, which result from the approximation of the beamwise variations being neglected. Mathematically, the flat ship model is however more difficult to deal with than the thin ship model in that a mixed boundary value problem must be solved therein.

Most of the authors of the references mentioned above considered the steady (that is, large time) problem, and as a consequence, the boundary conditions in the far field, the so-called radiation conditions, are yet to be justified. Recently, even the attainability of a steady state was questioned by Vanden-Broeck et al. ([26]). Based on a small

\footnotetext{
${ }^{1}$ School of Mathematics and Applied Statistics, The University of Wollongong, Wollongong NSW 2522, Australia; e-mail: spz@uow.edu.au.

${ }^{2}$ Center for Coastal and Land-Margin Research, Department of Environmental Science and Engineering, Oregon Graduate Institute of Science and Technology, Beaverton OR 97006, USA.

(C) Ausiralian Mathematical Society 2003, Serial-fee code 1446-1811/03
} 
Froude number expansion, their numerical solutions suggest that there exists no wavefree solution for a two-dimensional semi-infinite ship in water of infinite depth. They therefore argued that the bow flow should be modelled as a jet rising along the bow and falling down onto the oncoming stream ([6]). Although they treated the spray as steady (for the sake of simplicity) under the assumption that the impact of the jet on the oncoming stream is small, their results actually imply that a steady bow flow is not possible; splash will generally occur. On the other hand, with careful numerical experiments, Grosenbaugh and Yeung ([10]) conjectured that a steady bow flow with a stagnation point over the bow (see, for example, [5]) was possible at small Froude numbers. However, due to a numerical difficulty associated with the increasingly higher wavenumber near the bow (which is commonly present in small-time solutions for a moving object that intersects a free surface; see Roberts [20]), they were unable to offer conclusive evidence on this issue.

Besides the steady models, small-time perturbation models have also been explored $([13,14,20])$. These models are widely used to study the small-time evolution of the free surface when a surface-piercing plate extending to the bottom of the water is moving at a constant or varying speed. Mathematically simple as it is, this example is however inappropriate for large-time analysis since the water is continuously accumulated in front of the plate which eventually leads to the formation of a jet there and thus nonlinearity becomes dominant at large time, as commented in Joo et al. ([13]).

So far the two-dimensional ship problem has been solved predominantly using numerical methods such as the integral equation methods (for example [4, 21, 22] etc.). Owing to the wild behaviour of the kernels, the numerical approaches employed to solve these integral equations suffer severely from convergence problems ([2]), and therefore the relationship between the hull shape and the flow quantities is by no means clear. On the other hand, analytical results would certainly help numerical model developers, for example, to estimate the scales of certain parameters which are undoubtedly needed in a sophisticated numerical model ([2]).

In this paper, a linear analytical model for a two-dimensional flat ship is considered. The ship is modelled as a semi-infinite object in order to study the bow and stern flows separately. In literature, whether or not the boundary conditions can be linearised used to be controversial. But according to Haussling [11] and Fernandez ([8]), the linearisation seems to be valid in the far field for relatively large Froude numbers. In particular, Haussling ([11]) compared the results from the fully nonlinear potential theory and a linear flat ship theory and found a broad agreement between the two for draught-based Froude numbers greater than 3. In this paper, we are mainly concerned with the far-field solution. However, if the evolution of bow waves is to be studied, which inevitably involves the near-field solution, a matched asymptotic analysis can be employed and this is left for future study.

The plan of this paper is as follows. We first show, using a transient model, 
that a steady state is attainable at the large-time limit. We shall also show that a serious deficiency of the widely used small Froude number expansion $([5,26])$ is that it does not discriminate between the bow and stern flows even in transient states. Although our transient solution is given in a closed form, it is so involved that useful information can hardly be inferred from it. However, in addition to serving as a proof of the existence of the steady-state solution, the formal analytical solution for transient states also supplies the radiation condition needed in the steady problem. Then we solve the steady problem with the Wiener-Hopf technique and obtain closed-form farfield results for an arbitrary hull shape. The Wiener-Hopf technique has been widely used to solve various mixed boundary value problems occurring in electromagnetics, acoustics, elasticity etc. $([3,12,18])$. Finally, the relationship between the hull shape and the lee wave response is discussed with the results of several specific examples. In particular, we show that the problem of finding the optimal ship hull shape, which has been a very challenging numerical task (see, for example, $[7,15]$ ), is reduced to that of no more than minimising a functional. The optimal shape obtained in this way can serve as a useful first guide in engineering designs.

\section{Transient problems}

We assume that the fluid is inviscid, incompressible and infinitely deep. But the following analysis can be easily extended to the case of finite depth. A Cartesian coordinate system $x$ oy with the $x$-axis pointing to the right and the $y$-axis pointing upwards is attached to the moving ship, which is modelled as a semi-infinite object extending from $x=-\infty$ to $x=0$. The ship and the fluid are motionless for $t<0$ and at $t=0^{+}$the ship starts to move at a constant speed $U$, where $U>0(<0)$ corresponds respectively to a bow (stern) flow problem.

By choosing the length and velocity scales respectively as $U^{2} / g$ and $|U|$, where $g$ is the gravitational acceleration, the non-dimensionalised governing equation and boundary and initial conditions are given by

$$
\begin{aligned}
& \nabla^{2} \Phi=0, \quad \text { in the fluid region, } \\
& \Phi_{y}=\epsilon f^{\prime}\left(\lambda-\Phi_{x}\right), \quad y=-\epsilon f(x), \quad x<0, \\
& \left.\begin{array}{rl}
\Phi_{y} & =\breve{\eta}_{t}+\left(\Phi_{x}-\lambda\right) \breve{\eta}_{x}, \\
0 & =\breve{\eta}+\Phi_{t}-\lambda \Phi_{x}+\left(\Phi_{x}^{2}+\Phi_{y}^{2}\right) / 2,
\end{array}\right\} \quad y=\check{\eta}(x, t), x>0, \\
& \nabla \Phi \rightarrow 0, \quad r=\sqrt{x^{2}+y^{2}} \rightarrow \infty, \\
& \left.\Phi\right|_{y=0}=\left.\Phi_{t}\right|_{y=0}=\check{\eta}=0, \quad t=0, x>0 \text {, }
\end{aligned}
$$

where $\nabla$ is the two-dimensional gradient operator, subscripts denote partial differentiation, $\Phi$ is the velocity potential, the ship hull is given by $y=-\epsilon f(x)$ with $\epsilon$ 
being the dimensionless draught which is assumed to be small in the current flat ship theory, $\lambda= \pm 1$ corresponds respectively to bow and stern flows, and $y=\breve{\eta}(x, t)$ is the instantaneous location of the free surface. The origin of the coordinate system is located at the intersection between the hull and the undisturbed free surface so that $f(0)=0$. Other geometrical restrictions on $f(x)$ are that $f(x)=O(1)$, for $x<0$, and $f(x) \rightarrow 1, f^{\prime}(x), f^{\prime \prime}(x) \ldots \rightarrow 0$ as $x \rightarrow-\infty$, that is, the hull is virtually flat in the far field.

We then linearise the problem by assuming $\epsilon \ll 1$ and expanding the unknowns as

$$
\begin{aligned}
& \Phi=\epsilon \phi+O\left(\epsilon^{2}\right), \\
& \check{\eta}=\epsilon \eta+O\left(\epsilon^{2}\right) .
\end{aligned}
$$

Then the linearised differential system becomes

$$
\begin{gathered}
\nabla^{2} \phi=0, \quad \text { in the fluid region, } \\
\phi_{y}=\lambda f^{\prime}, \quad y=0, x<0, \\
\left.0=\phi_{y}+\phi_{t t}-2 \lambda \phi_{x t}+\phi_{x x},\right\} y=0, x>0, \\
\eta=\lambda \phi_{x}-\phi_{t}, \\
\nabla \phi \rightarrow 0, \quad r=\sqrt{x^{2}+y^{2}} \rightarrow \infty, \\
\left.\phi\right|_{y=0}=\left.\phi_{t}\right|_{y=0}=\eta=0, \quad t=0, x>0,
\end{gathered}
$$

which is a mixed initial-boundary value problem.

Before we proceed to solving the differential system (2.1)-(2.5) using integral transformations and the Wiener-Hopf technique, it is advantageous to examine the relationship between the bow and stern flows. It can be readily verified from the above differential system that the relationship

$$
\phi_{s}=-\phi_{b}, \quad x_{s}=-x_{b},
$$

holds, where the subscripts " $s$ " and " $b$ " denote the variables used respectively in "stern" and "bow" flow cases. That is, the two problems are equivalent provided that the solutions for $x>0$ and $x<0$ are interchanged. Therefore we shall only discuss the bow flow case and set $\lambda=1$ from here on. It must be noted that such a reversibility between the "stern" and "bow" flows is consistent with one's expectation because reversing the direction of the ship's motion is equivalent to interchanging downstream and upstream fluid motions. On the other hand, it can be shown that the small Froude number expansion leads to a series of linear problems in which bow and stern flows are completely equivalent at any order. Interestingly, with this analysis on transients, the reason why only one kind of steady flow was obtained in Vanden-Broeck et al. ([26]) manifests itself; the radiation conditions for both bow 
and stern flows are identical to each other and therefore the steady-state solution, if it exists, is either wave-like or wave-free. Apart from the undesirable divergence of the small Froude number expansion ([26]), this non-discrimination between the two flows is the main reason why we prefer not to use the small Froude number expansion here. Although the current flat ship model does not suffer from these deficiencies, it is at the cost of solving a much more difficult mixed boundary value problem.

To solve the differential system (2.1)-(2.5), we take the Laplace transform of the unknowns

$$
\hat{\phi}(x, y, s)=\int_{0}^{\infty} \phi(x, y, t) e^{-s t} \mathrm{~d} t,
$$

and the transformed system becomes

$$
\begin{aligned}
& \nabla^{2} \hat{\phi}=0, \quad \text { in the fluid region, } \\
& \hat{\phi}_{y}=\frac{1}{s} f^{\prime}, \quad y=0, x<0, \\
& \left.\begin{array}{l}
0=\hat{\phi}_{y}+s^{2} \hat{\phi}-2 s \hat{\phi}_{x}+\hat{\phi}_{x x}, \\
\hat{\eta}=\hat{\phi}_{x}-s \hat{\phi},
\end{array}\right\} \quad y=0, x>0, \\
& \nabla \hat{\phi} \rightarrow 0, \quad r=\sqrt{x^{2}+y^{2}} \rightarrow \infty \text {. }
\end{aligned}
$$

Then we take the Fourier transform of $\hat{\phi}$ with respect to $x$ :

$$
\psi(\kappa, y, s)=\frac{1}{\sqrt{2 \pi}} \int_{-\infty}^{+\infty} \hat{\phi}(x, y, s) e^{-i \kappa x} \mathrm{~d} x
$$

By virtue of the Laplace equation and the boundary condition (2.8), $\psi$ must be of the form

$$
\psi=A(\kappa) e^{|\kappa| y}
$$

where $A(\kappa)$ is to be determined. Therefore we have the formal solution:

$$
\hat{\phi}=\frac{1}{\sqrt{2 \pi}} \int_{-\infty}^{+\infty} A(\kappa) e^{|\kappa| y} e^{i \kappa x} \mathrm{~d} \kappa
$$

with $A(\kappa)$ to be found by satisfying the remaining boundary conditions (2.6) and (2.7).

As one expects, the perturbation near the origin is non-uniform. Strictly speaking, a matched asymptotic analysis must be used to find the near-field (inner) solution ([27]). An inner solution is necessary if the evolution of the bow waves is of concern. Since in this paper we are mainly interested in the outer (far-field) solution, we shall not pursue an inner solution here. Research on the inner solution is continuing and the results will be reported elsewhere. 
Even without the need to find the near-field solution, the singularity at the origin hinders the Fourier transform from being applied to the free-surface boundary condition. To circumvent this difficulty, we adopt a dual integral equation method (see, for example, [18]). Substituting the formal solution (2.9) into the boundary conditions at $y=0$ yields a pair of integral equations to be satisfied on the two parts of the boundary:

$$
\begin{array}{ll}
\frac{1}{\sqrt{2 \pi}} \int_{-\infty}^{+\infty} B(\kappa) e^{i \kappa x} \mathrm{~d} \kappa=\frac{1}{s} f^{\prime}(x), & x<0, \\
\frac{1}{\sqrt{2 \pi}} \int_{-\infty}^{+\infty} B(\kappa) G(\kappa) e^{i \kappa x} \mathrm{~d} \kappa=0, & x>0,
\end{array}
$$

where $B(\kappa)=|\kappa| A(\kappa)$ and $G(\kappa)=1+(s-i \kappa)^{2} /|\kappa|$. Since $|G(\kappa)| \sim|\kappa|$ as $|\kappa| \rightarrow \infty$, this is a special case discussed in Noble ([18, page 222]). To solve the above dual integral equations, we split $G(\kappa)$ in a standard Wiener-Hopf fashion:

$$
G(\kappa)=-\sqrt{\kappa}_{+} \sqrt{\kappa}_{-} L_{+}(\kappa) L_{-}(\kappa) \equiv G_{+}(\kappa) G_{-}(\kappa)
$$

where $G_{ \pm}(\kappa)=\mp \sqrt{\kappa}_{ \pm} L_{ \pm}(\kappa)$. Hereafter we use " + " and "-" to denote that the function subscriptised is analytical on the upper and lower half of the complex $\kappa$-planes respectively. Here $L_{ \pm}(\kappa)$ is the Wiener-Hopf decomposition of $L(\kappa)=-1 /|\kappa|-$ $(s-i \kappa)^{2} / \kappa^{2}$ and can be found in a standard way:

$$
\ln L_{ \pm}(\kappa)= \pm \frac{1}{2 \pi i} \int_{\Gamma_{ \pm}} \frac{\ln L(\zeta)}{\zeta-\kappa} \mathrm{d} \zeta,
$$

where the contours $\Gamma_{ \pm}$are defined in Noble ([18, page 13]). The multi-valued functions (such as $\sqrt{\kappa}_{ \pm}$) are analytical once proper branch cuts are inserted into the complex plane. In this paper, we take the negative real axis as the branch cut. It should also be noted that $G_{ \pm}(\kappa)$ and $L_{ \pm}(\kappa)$ are non-zero on the respective half-planes ([18, page 15$\left.]\right)$ but may have zeros on the real axis.

Changing $x$ to $x-\xi$ in (2.10) and to $x+\xi$ in (2.11) with $\xi>0$, and then multiplying (2.10) and $(2.11)$ by $(1 / \sqrt{2 \pi}) M_{\mp}(\xi)$ respectively, and finally integrating both sides with respect to $\xi$ from 0 to $+\infty$ gives

$$
\begin{aligned}
& \frac{1}{\sqrt{2 \pi}} \int_{-\infty}^{+\infty} N_{-}(\kappa) B(\kappa) e^{i \kappa x} \mathrm{~d} \kappa=\frac{1}{\sqrt{2 \pi} s} \int_{0}^{+\infty} M_{-}(\xi) f^{\prime}(x-\xi) \mathrm{d} \xi, \quad x<0, \\
& \frac{1}{\sqrt{2 \pi}} \int_{-\infty}^{+\infty} N_{+}(\kappa) B(\kappa) G(\kappa) e^{i \kappa x} \mathrm{~d} \kappa=0, \quad x>0,
\end{aligned}
$$

where

$$
N_{ \pm}(\kappa)=\frac{1}{\sqrt{2 \pi}} \int_{-\infty}^{+\infty} M_{ \pm}(\xi) e^{ \pm i \kappa \xi} \mathrm{d} \xi
$$


are respectively analytical and vanish as $|\kappa| \rightarrow \infty$ on the upper and lower half planes. Following Noble, we take

$$
N_{-}(\kappa)=\frac{G_{-}(\kappa)}{\kappa-\kappa_{2}}, \quad N_{+}(\kappa)=\frac{1}{G_{+}(\kappa)},
$$

where $\kappa_{2}$ is an arbitrary number on the upper half plane $\left(\operatorname{Im}\left\{\kappa_{2}\right\}>0\right)$. From (2.14) $M_{ \pm}(\xi)$ can be determined by the inverse Fourier transform

$$
M_{ \pm}(\xi)=\frac{1}{\sqrt{2 \pi}} \int_{-\infty}^{+\infty} N_{ \pm}(\kappa) e^{\mp i \kappa \xi} \mathrm{d} \kappa
$$

It can be shown via a contour integration that $M_{ \pm}(\xi)=0$ for $\xi<0$.

Substituting (2.15) into the dual integral equations (2.12) and (2.13) gives

$$
\begin{aligned}
& \frac{1}{\sqrt{2 \pi}} \int_{-\infty}^{+\infty} \frac{G_{-}(\kappa)}{\kappa-\kappa_{2}} B(\kappa) e^{i \kappa x} \mathrm{~d} \kappa=\frac{1}{\sqrt{2 \pi} s} \int_{0}^{+\infty} M_{-}(\xi) f^{\prime}(x-\xi) \mathrm{d} \xi, \quad x<0 \\
& \frac{1}{\sqrt{2 \pi}} \int_{-\infty}^{+\infty} G_{-}(\kappa) B(\kappa) e^{i \kappa x} \mathrm{~d} \kappa=0, \quad x>0 .
\end{aligned}
$$

Multiplying the first equation by $e^{-i k_{2} x}$ and then differentiating it with respect to $x$ yields

$$
\begin{aligned}
& \frac{1}{\sqrt{2 \pi}} \int_{-\infty}^{+\infty} G_{-}(\kappa) B(\kappa) e^{i \kappa x} \mathrm{~d} \kappa \\
& \quad=-\frac{i}{\sqrt{2 \pi} s} e^{i \kappa_{2} x} \frac{\mathrm{d}}{\mathrm{d} x} e^{-i \kappa_{2} x} \int_{0}^{+\infty} M_{-}(\xi) f^{\prime}(x-\xi) \mathrm{d} \xi, \quad x<0
\end{aligned}
$$

The Fourier inversion of (2.16) and (2.17) leads to

where

$$
G_{-}(\kappa) B(\kappa)=-\frac{i}{2 \pi s} \int_{-\infty}^{0} X(x) e^{-i \kappa x} \mathrm{~d} x \equiv \frac{I(\kappa, s)}{s},
$$

$$
X(x)=e^{i \kappa_{2} x} \frac{\mathrm{d}}{\mathrm{d} x} e^{-i \kappa_{2} x} \int_{0}^{+\infty} M_{-}(\xi) f^{\prime}(x-\xi) \mathrm{d} \xi
$$

We then have the formal solution as

$$
\eta(x, t)=\left.\left(\phi_{x}-\phi_{t}\right)\right|_{y=0}=\frac{1}{2 \pi i \sqrt{2 \pi}} \int_{\Upsilon} \frac{e^{s t}}{s} \mathrm{~d} s \int_{-\infty}^{+\infty} \frac{I(\kappa, s)}{|\kappa| G_{-}(\kappa, s)} e^{i \kappa x}(i \kappa-s) \mathrm{d} \kappa,
$$

where $\Upsilon$ is the contour for the inverse Laplace transform and is located to the right of all singularities (including poles, branch points and essential singularities). It should 
be noted that we have been using $G_{-}(\kappa)$ etc. as shorthand notation for $G_{-}(\kappa, s)$ etc. for brevity, but from now on we shall use the full notation.

The large time asymptotic form of the solution crucially depends on the poles and possible branch points on the complex $s$-plane. By definition, $G(\kappa, s)$ has two zeros at $s=i \kappa \pm i \sqrt{|\kappa|}$, which are pure imaginary numbers. Thus we can choose the contour $\Upsilon$ as the imaginary axis with a proper indentation around the poles or branch points. From (2.15), $G_{+}(\kappa, s)$ cannot have zeros and therefore $G_{-}(\kappa, s)$ must have simple zeros at $s=i \kappa \pm i \sqrt{|\kappa|}$. So the solution is in the form

$$
\Omega=\int_{-\infty}^{+\infty} e^{i \kappa x} \mathrm{~d} \kappa \int_{\Upsilon} \frac{e^{s t}}{s\left(s-i b_{1}\right)\left(s-i b_{2}\right)} F(\kappa, s) \mathrm{d} s
$$

where $F(\kappa, s)$ is analytical on the left half $s$-plane, and $b_{1,2}=\kappa \pm \sqrt{|\kappa|}$. From the residue theorem, we have

$$
\Omega=-2 \pi i \int_{-\infty}^{+\infty}\left[\frac{F\left(\kappa, i b_{1}\right) e^{i b_{1} \ell}}{b_{1}\left(b_{1}-b_{2}\right)}+\frac{F\left(\kappa, i b_{2}\right) e^{i b_{2} t}}{b_{2}\left(b_{2}-b_{1}\right)}+\frac{F(\kappa, 0)}{b_{1} b_{2}}\right] e^{i \kappa x} \mathrm{~d} \kappa,
$$

with the understanding that the path of integration is curved (from either above or below) around the poles on the real axis. The last term in (2.19) represents a steady state. As $t \rightarrow \infty$, the first and second term vanish like $t^{-1 / 2}$ by the method of stationary phase (see, for example, [23]), provided that the indentations around these poles are all from above (otherwise they would give rise to some oscillatory components as $t \rightarrow \infty$ ). The justification of this particular choice of the indentation is as follows. If we had chosen to curve the path around the poles from below, we would have ended up with a situation where a train of stationary waves and some propagating waves appear far upstream (in a bow flow problem) at large time. That is, we would have obtained a quasi-steady solution. But this is impossible since the group velocity of the surface waves is less than the phase velocity and therefore the wave energy cannot be radiated to the far upstream.

Upon the establishment of the attainability of a steady state, the radiation condition follows immediately. Since the path of integration in (2.19) is curved around the poles from above, it can be shown that $\eta \rightarrow 0$ as $x \rightarrow+\infty$ (far upstream) in a steady problem.

So far we have obtained a closed-form solution for the transient states. Such a transient solution constructed with the Wiener-Hopf technique is undoubtedly of great theoretical significance, though extracting further information for transient solutions remains a very difficult task, which is of little interest to us at this stage anyway; a further analysis on the transient part (for example, for transient bow wave problems) is left for future studies. So we now turn to the steady problem for a detailed analysis. 


\section{Steady-state problems}

The dual integral equations and the formal solution corresponding to the steady state are

$$
\begin{array}{cc}
\frac{1}{\sqrt{2 \pi}} \int_{-\infty}^{+\infty} A(\kappa)|\kappa| e^{i \kappa x} \mathrm{~d} \kappa=f^{\prime}(x), & x<0 \\
\frac{1}{\sqrt{2 \pi}} \int_{-\infty}^{+\infty} A(\kappa)|\kappa| G(\kappa) e^{i \kappa x} \mathrm{~d} \kappa=0, & x>0 \\
\eta=\frac{i}{\sqrt{2 \pi}} \int_{-\infty}^{+\infty} A(\kappa) \kappa e^{i \kappa x} \mathrm{~d} \kappa &
\end{array}
$$

where $G(\kappa)=|\kappa|-1$. As explained before, we are mainly interested in the farfield solution, which is crucially dependent on the zeros of $G_{-}(\kappa)$ (see, for example, (2.18)). Instead of directly decomposing $G(\kappa)$ and trying to find the zeros of $G_{-}(\kappa)$, we adopt here a much less laborious approach. Differentiating (3.1) with respect to $x$ twice and adding the resultant equation to (3.1) leads to

$$
\frac{1}{\sqrt{2 \pi}} \int_{-\infty}^{+\infty} A(\kappa)|\kappa|\left(\kappa^{2}-1\right) e^{i \kappa x} \mathrm{~d} \kappa=-f^{\prime}(x)-f^{\prime \prime \prime}(x), \quad x<0 .
$$

We then proceed as in the previous section and obtain

$$
\begin{aligned}
& \frac{1}{\sqrt{2 \pi}} \int_{-\infty}^{+\infty} N_{-}(\kappa) C(\kappa) K(\kappa) e^{i \kappa x} \mathrm{~d} \kappa \\
& \quad=-\frac{1}{\sqrt{2 \pi}} \int_{0}^{+\infty} M_{-}(\kappa)\left[f^{\prime}(x-\xi)+f^{\prime \prime \prime}(x-\xi)\right] \mathrm{d} \xi, \quad x<0, \\
& \frac{1}{\sqrt{2 \pi}} \int_{-\infty}^{+\infty} N_{+}(\kappa) C(\kappa) e^{i \kappa x} \mathrm{~d} \kappa=0, \quad x>0,
\end{aligned}
$$

where $C(\kappa)=A(\kappa)|\kappa|(|\kappa|-1), K(\kappa)=1+|\kappa|$, and $N_{ \pm}(\kappa)$ are defined in (2.14). Unlike in the previous section, this time we take

$$
N_{+}(\kappa)=\frac{K_{+}(\kappa)}{\kappa-\kappa_{3}}, \quad N_{-}(\kappa)=\frac{1}{K_{-}(\kappa)},
$$

with $\operatorname{Im}\left\{\kappa_{3}\right\}<0$. The Wiener-Hopf decomposition of $K(\kappa)$ for real $\kappa$ can be found as (see, for example [3, Equation (A.6)])

$$
\begin{aligned}
K_{+}(\kappa) & =\exp \left\{\frac{i}{\pi} \int_{0}^{\kappa} \frac{1}{1-u^{2}}\left[\frac{i u \pi}{2}+\ln (-i u)\right] \mathrm{d} u\right\} \\
& =\sqrt{1+|\kappa|} \exp \left\{\frac{i}{\pi} \int_{0}^{\kappa} \frac{\ln |u|}{1-u^{2}} \mathrm{~d} u\right\}
\end{aligned}
$$


Note that an arbitrary constant can be put into $K_{ \pm}(\kappa)$ with no effect on the final solution.

So the final solution reads

$$
\begin{aligned}
\eta & =\frac{i}{\sqrt{2 \pi}} \int_{-\infty}^{+\infty} \operatorname{sgn} \kappa \frac{I(\kappa)}{(|\kappa|-1) K_{+}(\kappa)} e^{i \kappa x} \mathrm{~d} \kappa \\
& =-\sqrt{\frac{2}{\pi}} \operatorname{Im}\left\{\int_{0}^{+\infty} \frac{I(\kappa)}{(\kappa-1) K_{+}(\kappa)} e^{i \kappa x} \mathrm{~d} \kappa\right\} \\
I(\kappa) & =-\frac{1}{2 \pi} \int_{-\infty}^{0} X(x) e^{-i \kappa x} \mathrm{~d} x \\
X(x) & =\int_{0}^{+\infty} M_{-}(\xi)\left[f^{\prime}(x-\xi)+f^{\prime \prime \prime}(x-\xi)\right] \mathrm{d} \xi \\
M_{-}(\xi) & =\frac{1}{\sqrt{2 \pi}} \int_{-\infty}^{+\infty} \frac{1}{K_{-}(u)} e^{i u \xi} \mathrm{d} u=\frac{1}{\sqrt{2 \pi}} \int_{-\infty}^{+\infty} \frac{K_{+}(u)}{1+|u|} e^{i u \xi} \mathrm{d} u .
\end{aligned}
$$

In (3.3), we have utilised a property of $K_{+}(\kappa)$, that is, $K_{+}(-\kappa)=\overline{K_{+}(\kappa)}$ (see, for example, (3.2)). Note that although $\eta$ is only defined for $x>0$ in a bow flow problem, we can extend it to $x<0$ to cover the stern flow due to the equivalence between the two flows. We already know from the radiation condition that $\eta \rightarrow 0$ as $x \rightarrow+\infty$ for bow flows. This amounts to curving the path around the pole at $\kappa=1$ from above in (3.3). Using Cauchy's residue theorem, one can show that for stern flows

$$
\eta_{s} \sim 2 \sqrt{2 \pi} \operatorname{Re}\left\{e^{-i x} \frac{I(1)}{K_{+}(1)}\right\}
$$

in the far field. This represents nothing but the stationary lee waves for stern flows. The right-hand side of (3.4) can be simplified as

$$
\begin{aligned}
K_{+}(1) & =\sqrt{2} e^{-i \pi / 8} \\
I(1) & =-\frac{1}{2 \pi} \int_{0}^{+\infty} M_{-}(\xi) \mathrm{d} \xi \int_{-\infty}^{0} e^{-i x}\left[f^{\prime}(x-\xi)+f^{\prime \prime \prime}(x-\xi)\right] \mathrm{d} x \\
& =-\frac{1}{2 \pi} \int_{0}^{+\infty} M_{-}(\xi)\left[f^{\prime \prime}(-\xi)+i f^{\prime}(-\xi)\right] \mathrm{d} \xi
\end{aligned}
$$

Equations (3.4)-(3.6) give an analytical form of the lee waves for an arbitrary hull shape. The details of numerical evolution of the integral in (3.6) can be found in the Appendix. It is interesting to note that another restriction on the function $f(x)$ is that $\alpha>3 / 2$ or $\alpha=1$ if $f(x) \sim(-x)^{\alpha}$ as $x \rightarrow 0^{-}$(see, for example, the Appendix); solutions for $0<\alpha<1$ and $1<\alpha \leq 3 / 2$ are unbounded. 


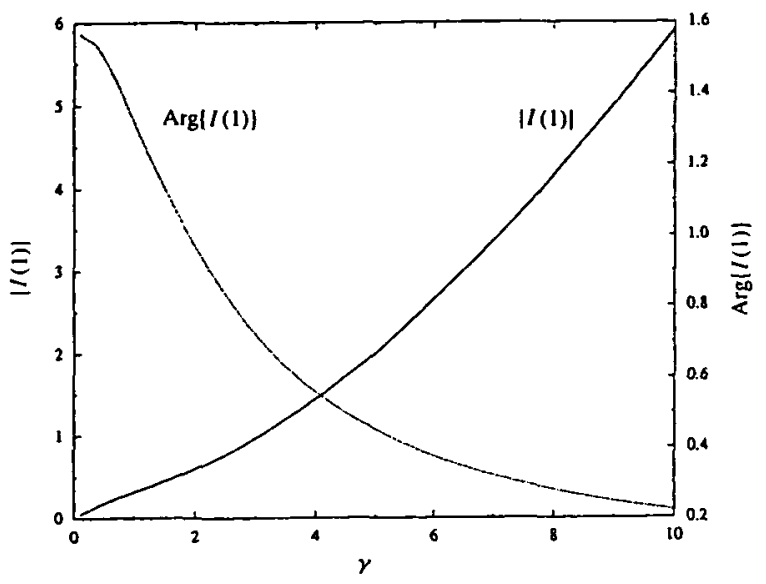

FIGURE 1. Magnitude and phase of $I(1)$ as a function of the slope.

As an example, we computed a simple hull profile of a flat-bottom ship terminated by a slope $\gamma$. The corresponding $f(x)$ is

$$
f(x)= \begin{cases}-\gamma x, & -1 / \gamma \leq x \leq 0 \\ 1, & x<-1 / \gamma\end{cases}
$$

and the magnitude and phase of $I(1)$ are shown in Figure 1. As the slope $\gamma$ approaches zero, the wave amplitude diminishes as expected. On the other hand, as the slope becomes large, the amplitude increases unboundedly. This simply indicates that the flat ship theory cannot handle an abrupt change in the hull profile. However, it implies that ships with such hulls would generally induce much larger drag. It is also seen from Figure 1 that the phase of the lee waves approaches a constant (zero in this case) as the slope increases.

In a numerical study of ship stern waves, Haussling ([11]) compared results from the fully nonlinear potential theory and a linear flat ship theory and found that the nonlinear effects are negligible for most practical purposes when draught-based Froude numbers $F=U / \sqrt{g d}$ are greater than 3 . For $F=3$ and the curved hull discussed in his paper,

$$
f(x)= \begin{cases}-\sin \left(F^{2} x / 9\right), & -4.5 \pi / F^{2} \leq x \leq 0 \\ 1, & x<-4.5 \pi / F^{2}\end{cases}
$$

his numerical result for the stern wave amplitude is $1.3 d$, which is in good agreement with our result $1.1 d$. After plotting out the free-surface displacement for this hull shape (Figure 3), we also compared the total pressure (which is the sum of hydrostatic 


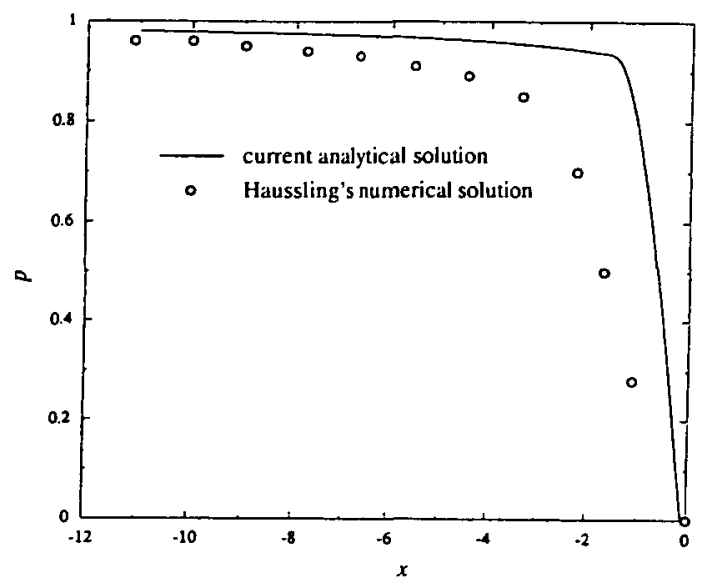

FIGURE 2. Comparison of the pressure distribution on a curved hull between Haussling's ([11]) numerical solution and current analytical solution for $F=3$.

and hydrodynamic pressures) distribution on the hull (Figure 2). As expected, the agreement is good in the far field $(x<-7)$ but poor in the near field, where the linearisation is not appropriate and a nonlinear analysis (possibly with the matched asymptotic method) is necessary. A weak singularity was also found at $x=0$. The hydrodynamic pressure was found to be negative, which results in a "sinkage" force discussed in Tuck ([24]).

One of the most fascinating questions associated with the bow and stern flows is the possible existence of a ship which experiences no drag ([7] and the references cited therein). With the current analytical solution at hand, the problem is reduced to solving an integral equation $I(1)=0$, which is ill-posed as the solution is not unique. A more reasonable problem is to optimise the hull shape so that the ship experiences a minimum drag. This amounts to minimising the functional $|I(1)|$ subject to the boundary conditions $f(0)=0, f(x) \rightarrow 1, f^{\prime}(x), f^{\prime \prime}(x) \ldots \rightarrow 0$ as $x \rightarrow-\infty$. But this would lead to a hull with vanishingly small slope since we have shown that the amplitude diminishes as $\gamma \rightarrow 0$ (see, for example, Figure 1; this can also be proved strictly with the results in the Appendix). Of course, such a hull shape is unrealistic since the real hull is of finite length. But for a hull of large length, we can propose a more meaningful problem, that is, to minimise $|I(1)|$ subject to the boundary conditions $f(0)=0$ and $f(x)=1$ for $x \leq-a$ where $a$ is a pre-assigned value. This amounts to finding the optimal shape between $-a \leq x \leq 0$ with the hull being flat in $x<-a$. The optimal shape and minimum drag computed in this way will depend on the parameter $a$, which is in turn determined by some restrictions in design practice. Unfortunately, finding the optimal shape in this way remains a non-trivial task; numerical approaches seem to be inevitable. However, it does offer 


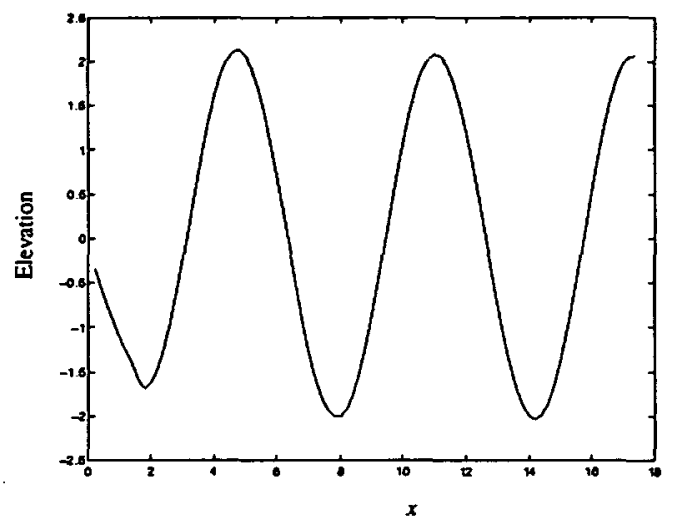

FIGURE 3. The corresponding free-surface displacement for $F=3$.

a very appealing and better approach to this very important practical problem, which is extremely difficult to solve using purely numerical methods; the optimal shape obtained in this way may serve as a useful first guide in engineering designs.

\section{Conclusions}

A flat ship theory was used to study ship stern and bow flows. The linear problem was solved analytically with the Wiener-Hopf technique. Upon starting from a general transient problem, a steady state proved to be attainable and the radiation conditions for the steady problem were also derived. The steady problem was then solved in detail and closed-form results were obtained for an arbitrary hull shape. Good agreements between the current analytical and previous numerical solutions were observed in the far field. Since the newly found analytical solution is for an arbitrary hull shape, we believe that it has shed some light on solving some previously very challenging problems such as the optimisation of hull shape.

Research is continuing on the evolution of bow waves and on finding the optimal hull shape by minimising a functional (where numerical approaches seem to be inevitable) and results will be reported in a forthcoming paper.

\section{Acknowledgements}

The second author would like to gratefully acknowledge financial support from DEET (Australia) for an Overseas Postgraduate Research Scholarship (OPRS) and from the University of Wollongong for a University of Wollongong Postgraduate Research Award (UPRA). 


\section{Appendix A: Numerical evaluation of (3.6)}

We first simplify $M_{-}(\xi)$ and separate the singularity out as

$$
\begin{aligned}
M_{-}(\xi)= & 2 \sqrt{\frac{2}{\pi}} \int_{0}^{\infty} \frac{\sin [-\pi / 8-H(u) / 2 \pi]}{\sqrt{1+u}} \sin \left[u \xi+\frac{H(u)}{2 \pi}-\frac{\pi}{8}\right] \mathrm{d} u \\
& -\frac{2}{\sqrt{\xi}}\left[\cos \left(\xi+\frac{\pi}{4}\right) C\left(\sqrt{\frac{2 \xi}{\pi}}\right)+\sin \left(\xi+\frac{\pi}{4}\right) S\left(\sqrt{\frac{2 \xi}{\pi}}\right)\right]+\sqrt{\frac{2}{\xi}} \cos \xi,
\end{aligned}
$$

where $C(x)$ and $S(x)$ are Fresnel integrals defined in Abramowitz and Stegun [1], $H(u)=\int_{0}^{u}(\ln v) /\left(1-v^{2}\right) d v \quad(u \geq 0)$ and only the last term possesses a weak singularity $(\sim 1 / \sqrt{\xi})$ at $\xi=0$. On account of this singularity, we obtain another restriction on the function $f(x)$, that is, if $f(x) \sim(-x)^{\alpha}$ as $x \rightarrow 0^{-}$, then $\alpha>3 / 2$ or $\alpha=1$. That is to say, the current model cannot handle an abrupt change at $x=0$ (with $\alpha<1$ ).

The functions $C(x)$ and $S(x)$ were computed using truncated series expansions ([1, page 301]). For large arguments, the asymptotic forms were used ([1, page 322]).

The function $H(u)$ was computed using the following two truncated series expansions depending on the value of the argument $u$ :

$$
H(u)=\left\{\begin{array}{lr}
\frac{1}{2} \ln u \ln \frac{1+u}{1-u}-\sum_{n=0}^{\infty} \frac{u^{2 n+1}}{(2 n+1)^{2}}, & 0 \leq u \leq 1 \\
\frac{1}{2} \ln u \ln \frac{u+1}{u-1}-\frac{\pi^{2}}{4}+\sum_{n=0}^{\infty} \frac{u^{-2 n-1}}{(2 n+1)^{2}}, & u \geq 1 .
\end{array}\right.
$$

In deriving the second expansion, the following integral was used ([9]):

$$
H(+\infty)=\int_{0}^{\infty} \frac{\ln v}{1-v^{2}} \mathrm{~d} v=-\frac{\pi^{2}}{4}
$$

For simple geometrical functions $f(x)$, it is usually possible to reduce the double integral in (3.6) to single integrals by exchanging the order of integration; otherwise the double integral has to be evaluated. The non-singular and Cauchy principle value integrals (which occurred in the computation of the pressure distribution in Figure 2) were evaluated with two computer programs DQAG and DQAWC from QUADPACK [19] which employ adaptive schemes.

\section{References}

[1] M. Abramowitz and I. A. Stegun, Handbook of mathematical functions (Dover, New York, 1970). 
[2] S. Cole, “A simple example from flat-ship theory", J. Fluid Mech. 189 (1988) 301-310.

[3] R. V. Craster and C. Atkinson, "Mixed boundary value problems in non-homogeneous elastic materials", Q. J. Mech. Appl. Math. 47 (1988) 183-206.

[4] E. Cumberbatch, "Two-dimensional planing at high Froude number", J. Fluid Mech. 4 (1958) 466-478.

[5] G. Dagan and M. P. Tulin, "Two-dimensional free-surface gravity flow past blunt bodies", J. Fluid Mech. 51 (1972) 529-543.

[6] F. Dias and J.-M. Vanden-Broeck, "Nonlinear bow flows with spray", J. Fluid Mech. 255 (1993) 91-102.

[7] D. E. Farrow and E. O. Tuck, "Further studies of stern wavemaking", J. Austral. Math. Soc. Ser. B 36 (1995) 424-437.

[8] G. Fernandez, "Nonlinearity of the three-dimensional flow past a flat blunt ship", J. Fluid Mech. 108 (1981) 345-361.

[9] I. S. Gradshteyn and I. M. Ryzhik, Table of integrals and products (Academic Press, New York, 1965).

[10] M. A. Grosenbaugh and R. W. Yeung, "Nonlinear free-surface flows at a two-dimensional bow", J. Fluid Mech. 209 (1989) 57-75.

[11] H. J. Haussling, "Two-dimensional linear and nonlinear stern waves", J. Fluid Mech. 97 (1980) 759-769.

[12] D. S. Jones, "A simplifying technique in the solution of a class of diffraction problems", $Q . J$. Math. 3 (1952) 189-196.

[13] S. W. Joo, W. W. Schultz and A. F. Messiter, "An analysis of the initial wavemaker problem", J. Fluid Mech. 214 (1990) 161-183.

[14] A. C. King and D. J. Needham, "The initial development of a jet caused by fluid, body and freesurface interaction: Part 1. A uniformly accelerating plate", J. Fluid Mech. 268 (1994) 89-101.

[15] M. A. Madurashinhe and E. O. Tuck, "Ship bows with continuous and splashless flow attachment", J. Austral.. Math. Soc. Ser. B 27 (1986) 442-452.

[16] H. Maruo, "High- and low-aspect ratio approximation of planing surfaces", Schiffstechnik 14 (1967) 57-64.

[17] J. H. Michell, "The wave-resistance of a ship", Phil. Mag. 45 (1898) 106.

[18] B. Noble, Methods based on the Wiener-Hopf technique for the solution of partial differential equations (Pergamon Press, 1958).

[19] R. Piessens, E. de Doncker-Kapenga, C. W. Überhuber and D. K. Kahaner, QUADPACK (Springer, 1983).

[20] A. J. Roberts, "Transient free-surface flows generated by a moving vertical plate", Q.J. Mech. Appl. Math. 40 (1987) 129-158.

[21] L. I. Sedov, Two-dimensional problems in hydrodynamics and aerodynamics (Wiley and Sons, 1965).

[22] H. B. Squire, "The motion of a simple wedge along the water surface", Proc. Roy. Soc. London A. 243 (1958) 48-64.

[23] J. J. Stoker, Water Waves (Wiley and Sons, 1958).

[24] E. O. Tuck, "Shallow-water flows past slender bodies", J. Fluid Mech. 26 (1966) 81-95.

[25] E. O. Tuck, "Low-aspect-ratio flat ship theory", J. Hydronaut. 9 (1975) 1-12.

[26] J.-M. Vanden-Broeck, L. W. Schwartz and E. O. Tuck, "Divergent low-Froude number series expansion of nonlinear free-surface flow problems", Proc. Roy. Soc. London A 361 (1978) 207224.

[27] M. Van Dyke, Perturbation methods in fluid mechanics (Academic Press, New York, 1964). 